\title{
Die Low-Cost-Hypothese. Ein empirischer Test am Beispiel der Befürwortung einer City-Maut
}

\author{
Fabian Thiel
}

Eingegangen: 26. November 2019 / Angenommen: 25. August 2020 / Online publiziert: 9. Oktober 2020 (C) Der/die Autor(en) 2020

Zusammenfassung Die Low-Cost-Hypothese (LCH) postuliert, dass der Effekt einer Einstellung auf einstellungskonformes Handeln mit steigenden Kosten sinkt. Tutić et al. (2017) formalisieren die theoretischen Implikationen der LCH mithilfe eines mikroökonomischen Modells. Sie veranschaulichen, dass sich die LCH anhand einer verbesserten Teststrategie bewährt, während sie, basierend auf der vormals üblichen Modellierung mittels der expliziten Aufnahme eines Interaktionsterms, scheitert. Die von ihnen präsentierten Beispiele erlauben jedoch nur einen eingeschränkten Test der LCH im weiteren Sinn eines Einkommenseffekts - nicht aber im engeren Sinn eines tatsächlichen Preiseffekts einstellungskonformen Handelns. Dieser Beitrag liefert eine wichtige Ergänzung, indem am Beispiel der Befürwortung einer City-Maut eine erste Prüfung der verbesserten Teststrategie der $\mathrm{LCH}$ im engeren Sinn vorgelegt wird. Datengrundlage ist ein faktorieller Survey (FS), in dem im Rahmen einer Bevölkerungsbefragung in München und vier Umlandgemeinden im Frühsommer 2018 mehr als 1300 Personen über 5300 fiktive Mautmodelle bewerteten. Kernbefund der vorliegenden Untersuchung ist, dass sich die LCH anhand der verbesserten Teststrategie für den betrachteten Anwendungsfall der Befürwortung einer City-Maut bewährt.

Schlüsselwörter Einstellungen · Umweltbewusstsein · Faktorieller Survey · Experiment · City-Maut 


\title{
The Low-Cost-Hypothesis. An Empirical Test Taking the Support for a City Toll as an Example
}

\begin{abstract}
The low-cost hypothesis ( $\mathrm{LCH}$ ) postulates that the effect of an attitude on attitude-conforming behavior decreases with rising costs of attitude conformity. Using a microeconomic model, Tutic et al. (2017) formalized the theoretical implications of the $\mathrm{LCH}$. They illustrated that the $\mathrm{LCH}$ can fail using the previously common approach involving the explicit inclusion of an interaction term, whereas it holds using the enhanced test strategy. However, the presented examples allow only a limited test of the $\mathrm{LCH}$ in the more general sense of an income effect and not in the narrower sense of an actual price effect of attitude-conforming behavior. This article provides an important supplement by presenting the first test of the improved test strategy of the $\mathrm{LCH}$ in the narrower sense, using support for a city toll as an example. The data are based on a factorial survey in which more than 1300 respondents rated more than 5300 fictitious toll models as part of a population survey in Munich and four surrounding municipalities in the early summer of 2018. According to the presented analysis of the support for a city toll, the LCH holds using the improved test strategy.
\end{abstract}

Keywords Attitudes · Environmental concern · Factorial survey · Experiment · City toll

\section{Einleitung}

Die Belastung mit Luftschadstoffen hat weitreichende gesundheitliche Folgen, die von verschiedenen Erkrankungen der Atemwege und des Herz-Kreislauf-Systems bis hin zu vorzeitigen Todesfällen reichen (beispielsweise Apte et al. 2018; Lelieveld et al. 2019; Pope und Dockery 2006). In den Jahren 2000-2015 ist die Konzentration für Luftschadstoffe wie Stickstoffdioxid $\left(\mathrm{NO}_{2}\right)$ und Feinstaub $\left(\mathrm{PM}_{2,5}\right)^{1}$ in deutschen Ballungsräumen zwar deutlich gesunken, die von der Weltgesundheitsorganisation erarbeiteten Wirkungsschwellen von $20 \mu \mathrm{g} / \mathrm{m}^{3}\left(\mathrm{NO}_{2}\right)$ bzw. $10 \mu \mathrm{g} / \mathrm{m}^{3}\left(\mathrm{PM}_{2,5}\right)$ im Jahresmittel werden jedoch nach wie vor an einer Vielzahl der Messstationen insbesondere in verkehrsnaher Lage überschritten (Umweltbundesamt 2017, S. 46f.). Gerade in innerstädtischen Lagen scheinen wesentliche Minderungen der Belastung ohne eine Reduktion des Straßenverkehrsaufkommens kaum erreichbar. Da die individuelle Verkehrsmittelwahl hauptsächlich von materiellen Faktoren wie der Verfügbarkeit eines Autos sowie der Kostenersparnis im Vergleich zur Nutzung anderer Verkehrsmittel abhängt, sind nennenswerte Verhaltensanpassungen ohne Veränderungen der (gesetzlichen) Rahmenbedingungen jedoch nicht zu erwarten (Preisendörfer 2000). Zudem bleibt offen, inwiefern sich ein entsprechendes Problembewusstsein und umweltorientierte Einstellungen in der Befürwortung politischer Maßnahmen zur Veränderung eben jener Rahmenbedingungen niederschlagen. Das gilt insbesondere vor

\footnotetext{
1 Als Feinstaub werden Staubpartikel mit einem aerodynamischen Durchmesser $<10 \mu m\left(\mathrm{PM}_{10}\right)$ sowie noch feinere Partikel mit einem aerodynamischen Durchmesser $<2,5 \mu \mathrm{m}\left(\mathrm{PM}_{2,5}\right)$ bezeichnet.
} 
dem Hintergrund, dass sich eine Veränderung der gesetzlichen Rahmenbedingungen möglicherweise direkt auf die Kosten individuellen Mobilitätsverhaltens auswirkt. $\mathrm{Zu}$ denken sei dabei neben generellen Fahrverboten für bestimmte Fahrzeugtypen (beispielsweise „Dieselfahrverbote“) etwa an die Einführung einer City-Maut. Es stellt sich also die Frage, wie umweltbezogene Einstellungen in Anbetracht individuell anfallender Kosten auf die Absicht, solche Maßnahmen zu befürworten, wirken. Ist die beabsichtigte Befürwortung umso größer, je ausgeprägter umweltbezogene Einstellungen, aber auch je geringer die aus einer Befürwortung resultierenden Kosten sind?

\subsection{Bisherige Forschung zur LCH}

Die Low-Cost-Hypothese (LCH) stellt einen verbreiteten Ansatz zur Erklärung des hier im Fokus stehenden Zusammenspiels von Einstellung, Kosten und Verhalten dar. Sie besagt, dass mit steigenden Kosten einstellungskonformen Handelns der Effekt der Einstellung auf das Verhalten sinkt. Im Anschluss an grundlegende Arbeiten von Diekmann und Preisendörfer $(1998,2003)$ wurde die LCH in einer Vielzahl empirischer Arbeiten getestet und immer wieder auch theoretisch diskutiert (etwa Best und Kroneberg 2012; Braun und Franzen 1995; Diekmann 1998; Keuschnigg und Kratz 2018). Umweltsoziologische Anwendungen umfassen dabei beispielsweise die monetäre Bewertung von Biodiversität in Wäldern (Liebe 2007), die Umstellung auf ökologische Landwirtschaft (Best 2008) oder die Nutzung von Ökostrom (Neumann und Mehlkop 2018). Eine ganze Reihe von Arbeiten befasst sich etwa mit der Beteiligung an Recycling (Best 2009a, 2009b; Best und Kneip 2011; Derksen und Gartrell 1993; Diekmann und Preisendörfer 1998, 2003; Keuschnigg und Kratz 2018; Schultz und Oskamp 1996). Darüber hinaus wurde die LCH auch an anderen Anwendungsfällen, wie etwa der Spendenbereitschaft an Hilfsorganisationen (Mayerl 2010), der Durchsetzung sozialer Normen (Rauhut und Krumpal 2008) oder beruflicher Umzugsentscheidungen in Paarbeziehungen (Auspurg et al. 2014) getestet.

Die breite empirische Rezeption der LCH hat jedoch eine ebenso umfangreiche Bandbreite teils widersprüchlicher empirischer Befunde hervorgebracht. Dabei orientieren sich die Arbeiten durchaus an der von Diekmann und Preisendörfer (2003) vorgeschlagenen Teststrategie, die neben den Haupteffekten die Aufnahme eines multiplikativen Terms zwischen der Einstellung und den Kosten einstellungskonformen Verhaltens vorsieht. Besonders deutlich wird die unbefriedigende Befundlage an den Arbeiten zur Beteiligung an Recycling. So stützen manche der Arbeiten die LCH (Derksen und Gartrell 1993; Diekmann und Preisendörfer 1998, 2003), während andere Studien keine Unterstützung für die LCH finden (Best 2009a, 2009b; Best und Kneip 2011; Schultz und Oskamp 1996).

Umso mehr sind Bestrebungen hervorzuheben, die durch die neuerliche Auseinandersetzung mit der theoretischen Herleitung der LCH sowie ihrer Modellierung in der empirischen Anwendung möglicherweise dazu beitragen, die uneinheitliche Befundlage aufzuklären. Keuschnigg und Kratz (2018) argumentieren etwa, dass die widersprüchlichen Ergebnisse durch unterschiedliche normative Erwartungen bedingt seien, die wiederum den Zusammenhang zwischen Einstellung und einstel- 
lungskonformem Verhalten moderieren. So finden sie den von der LCH postulierten Zusammenhang zwar für Recycling von Plastik, nicht jedoch für Recycling von Glas. Das führen sie darauf zurück, dass aufgrund unterschiedlicher normativer Erwartungen das Ausmaß des Umweltbewusstseins für verschiedene Wertstoffe nicht in gleichem Maße verhaltensrelevant ist. Während Recycling von Glas normativ gewissermaßen ,,selbstverständlich“ geworden ist (Einstellung und Kosten spielen kaum mehr eine Rolle), ist dies für Plastik nicht der Fall (die von der LCH postulierten Zusammenhänge bleiben für die Entscheidung über die Beteiligung an Recycling relevant). Andererseits mag dies möglicherweise auch an zum Teil falschen Schlussfolgerungen aufgrund bisher üblicher Spezifikationen der Modelle liegen, wie Best und Kroneberg (2012) für binäre sowie Tutić et al. (2017) für kontinuierliche Verhaltensvariablen argumentieren.

Die von Tutić et al. (2017) vorgelegte Herleitung der LCH aus einem mikroökonomischen Modell legt für den Fall kontinuierlicher Verhaltensvariablen überzeugend dar, dass von der Aufnahme eines multiplikativen Terms der Einstellung mit den Kosten einstellungskonformen Handelns abzusehen ist. ${ }^{2}$ Die von ihnen vorgeschlagene Modellierung bildet das Zusammenspiel von Einstellung und Kosten vielmehr implizit ab und sieht daher keine explizite Aufnahme eines solchen Interaktionsterms vor. Gegenüber der herkömmlichen Teststrategie zeichnet sich diese verbesserte Teststrategie insbesondere durch zwei wesentliche Vorteile aus. Erstens ist sie aufgrund ihrer Herleitung aus der Mikroökonomik theoretisch fundiert und zweitens erlaubt sie aufgrund der starken Formalisierung präzise Vorhersagen (s. Abschn. 2).

Tutić et al. (2017) zeigen zudem, dass die LCH anhand der herkömmlichen Teststrategie scheitern kann, während sie sich anhand der von ihnen vorgeschlagenen verbesserten Teststrategie bewährt. Dazu illustrieren sie die verbesserte Teststrategie anhand zweier Beispiele, testen dabei jedoch lediglich die LCH im weiteren Sinn einer beschränkten Ressourcenausstattung. Die Kostspieligkeit einstellungskonformen Handelns wird in den Beispielen also anhand des Einkommens operationalisiert. Mithin wird getestet, dass je geringer das Einkommen (und damit auch der finanzielle Handlungsspielraum) ist, desto geringer sei der Effekt der Einstellung. ${ }^{3}$ Die LCH im engeren Sinn sieht hingegen die explizite Modellierung der Kosten einstellungskonformen Handelns (so gesehen im Sinne eines Preises einstellungskonformen Handelns) jenseits des Einkommenseffekts vor. Wie die Autoren auch selbst betonen, scheinen beide Varianten der LCH inhaltlich bedeutsam. Nach bestem Wissen existiert jedoch bisher noch keine Prüfung der LCH im engeren Sinn eines Preiseffekts anhand der verbesserten Modellierung.

\footnotetext{
2 Best und Kroneberg (2012) kommen für den Fall binärer Verhaltensvariablen ebenfalls zu dem Schluss, dass zur Prüfung der LCH kein variablenspezifischer Interaktionsterm aus Einstellung und Kosten einstellungskonformen Verhaltens (,Einstellung $\times$ Kosten“) in das Modell aufgenommen werden sollte.

3 Die Autoren sprechen in diesem Zusammenhang auch von einer „Low-Income-Hypothese“, um deutlich zu machen, dass die Kostspieligkeit einstellungskonformen Handelns hier über die Höhe des verfügbaren Einkommens, nicht aber über die für einstellungskonformes Handeln anfallenden Kosten gemessen wird.
} 


\subsection{Der Beitrag eines neuerlichen Tests}

In der vorliegenden Arbeit wird die von Tutić et al. (2017) vorgeschlagene Modellierung auf den Anwendungsfall der Befürwortung einer City-Maut zur Verbesserung der Luftqualität in größeren Städten übertragen. Es wird der Frage nachgegangen, ob der Einfluss des Umweltbewusstseins auf die Absicht, eine City-Maut zu befürworten, mit steigenden Kosten der Maut abnimmt, wie es anhand der LCH zu vermuten wäre. Dazu werden Daten einer Bevölkerungsbefragung verwendet. Neben Fragen zu umweltrelevanten Einstellungen sowie zur individuellen Verkehrsmittelnutzung umfasste die Erhebung einen faktoriellen Survey zur Befürwortung einer City-Maut in München. Den Befragten wurden jeweils vier mögliche Mautmodelle in Form von Vignetten zur Bewertung vorgelegt, wobei relevante Eigenschaften (Dimensionen), wie etwa die Höhe der Mautgebühren oder der Grad der durch die Maut voraussichtlich erreichten Luftverbesserung, experimentell variiert wurden. Insgesamt liegen über 5000 Vignettenurteile von mehr als 1300 Befragten vor, auf deren Basis die im Kontext der LCH relevanten Zusammenhänge geprüft werden.

Damit wird in zweierlei Hinsicht an die bestehende Literatur zur LCH angeknüpft. Zum einen wird die LCH im engeren Sinn eines Preiseffekts einstellungskonformen Handelns einer ersten empirischen Prüfung anhand der verbesserten Teststrategie unterzogen. Zum zweiten ist der gewählte Anwendungsfall gerade aufgrund der Relevanz gesetzlicher Rahmenbedingungen für die Kosten, welche bei der Nutzung verschiedener Verkehrsmittel anfallen, aufschlussreich. So wird geprüft, inwiefern die Bereitschaft zur einstellungskonformen Gestaltung gesetzlicher Rahmenbedingungen auch dann besteht, wenn daraus im Rahmen der individuellen Verkehrsmittelwahl zusätzliche Kosten erwachsen. Dabei wird an die Folgerungen von Diekmann und Preisendörfer (2003, S. 468) zu den Auswirkungen veränderter gesetzlicher Rahmenbedingungen auf individuelle, einstellungsrelevante Verhaltensentscheidungen angeknüpft. Im Fokus stehen hier jedoch nicht die in Reaktion auf veränderte Rahmenbedingungen neu zu treffenden individuellen Verhaltensentscheidungen, sondern der vorgelagerte Schritt der Zustimmung zu derlei Gesetzesreformen selbst. Insofern stellt es auch keine Einschränkung dar, dass anstatt auf tatsächliches Mobilitätsverhalten auf die Befürwortung einer politischen Maßnahme abgezielt wird, die sich - sollte sie eingeführt werden - dauerhaft in tatsächlichen Kosten für bestimmte Mobilitätsformen niederschlägt. Vielmehr muss beachtet werden, dass die hier untersuchte abhängige Variable der „Befürwortung“ einer City-Maut als eine Verhaltensabsicht zu verstehen ist. Es handelt sich also nicht um tatsächliche Wahlentscheidungen, sondern um die Absicht, die zu bewertenden Mautmodelle (etwa im Rahmen einer politisch verbindlichen Bürgerbefragung auf Kommunalebene) entsprechend zu befürworten.

\section{Theorie}

Eine nur geringe Korrelation zwischen Einstellung und Verhalten ist ein häufig zu beobachtendes Phänomen. Zu dessen Erklärung wird, aufbauend auf grundlegende Arbeiten von Diekmann und Preisendörfer (1998, 2003), oftmals die LCH heran- 
gezogen. Sie besagt zunächst allgemein, dass der Einfluss der Einstellung auf das Verhalten mit steigenden Kosten einstellungskonformen Verhaltens sinkt. Während sich daraus die Richtung des vermuteten Zusammenhangs ergibt, ist jedoch keine Aussage über die funktionale Form des Zusammenhangs (also darüber, in welchem Maße der Einfluss der Einstellung bei steigenden Kosten sinkt) enthalten.

\subsection{Die mikroökonomische Modellierung der LCH}

Tutić et al. (2017) integrieren diese zunächst allgemein formulierte Hypothese unter Rückgriff auf Grundlagen der Mikroökonomie (vgl. beispielsweise Braun und Gautschi 2011; Mas-Collel et al. 1995; Varian 1992) in ein Modell der Nachfragetheorie. ${ }^{4}$ Dabei wird von Akteuren ausgegangen, die Präferenzen über mögliche Handlungsalternativen aufweisen. Die Auswahl zur Verfügung stehender Handlungsalternativen ist jedoch Restriktionen unterworfen. Akteure verhalten sich rational in dem Sinne, dass sie diejenige Handlungsalternative oder Kombination aus Handlungsalternativen wählen, die sie unter den gegebenen Restriktionen am höchsten bewerten (die ihnen mithin den größten Nutzen stiftet). Sofern die Präferenzen vollständig und transitiv sind, lässt sich die Präferenzordnung als eine ordinale Nutzenfunktion verstehen. Die im Rahmen der LCH relevanten Zusammenhänge lassen sich sodann mithilfe einer Cobb-Douglas-Nutzenfunktion darstellen, wobei die Stärke der Einstellung (,,attitude“) $\alpha$ als Exponent des Ausmaßes einstellungskonformen Handelns einbezogen wird und so intrinsische Motive $\mathrm{zu}$ einstellungskonformem Handeln zu reflektieren vermag. Der individuelle Nutzen setzt sich schließlich aus dem (jeweils kontinuierlich gemessenen) Ausmaß einstellungskonformen $\left(x_{a} \geq 0\right)$ und einstellungsirrelevanten Handelns $\left(x_{\neg a} \geq 0\right)$ zusammen. Letzteres umfasst sämtliche Handlungsweisen, die nicht als einstellungskonform angesehen werden können. Dabei sind neben explizit im Widerspruch zur Einstellung stehenden Verhaltensweisen auch solche gemeint, die gewissermaßen als ,neutral“ zu betrachten sind. Es wird nunmehr unterstellt, dass sich Akteure so verhalten, als ob sie diese Nutzenfunktion maximieren würden. Unter der Berücksichtigung, dass die Wahl der jeweiligen Handlungsalternative unter Restriktionen in Form eines begrenzten Einkommens $m>0$ sowie der Preise $p_{a}>0$ für einstellungskonformes und $p_{\neg a}>0$ für einstellungsirrelevantes Handeln getroffen wird, ergibt sich die Nachfragefunktion nach einstellungskonformem Handeln als

$$
x_{a}^{*}\left(\alpha, m, p_{a}\right)=\alpha \frac{m}{p_{a}},
$$

wobei $x_{a}^{*}$ das optimale Ausmaß einstellungskonformen Handelns bezeichnet. Anhand der Nachfragefunktion lassen sich die im Rahmen der LCH relevanten Zusammenhänge zwischen Einstellung, Kosten und der gewählten Handlungsalternative in der vermuteten Weise beschreiben. So ergibt sich ein positiver Effekt der Einstellung,

\footnotetext{
4 Die Autoren verweisen auch auf alternative Ansätze zur Formalisierung der LCH, wie sie von Diekmann (1998) und von Braun und Franzen (1995) vorgelegt wurden, die jedoch gewisse Schwächen aufweisen. An dieser Stelle wird lediglich das hier auch verwendete Modell von Tutić et al. (2017) skizziert. Für weitere Details sowie eine Diskussion der alternativen Ansätze siehe Tutić et al. (2017).
} 

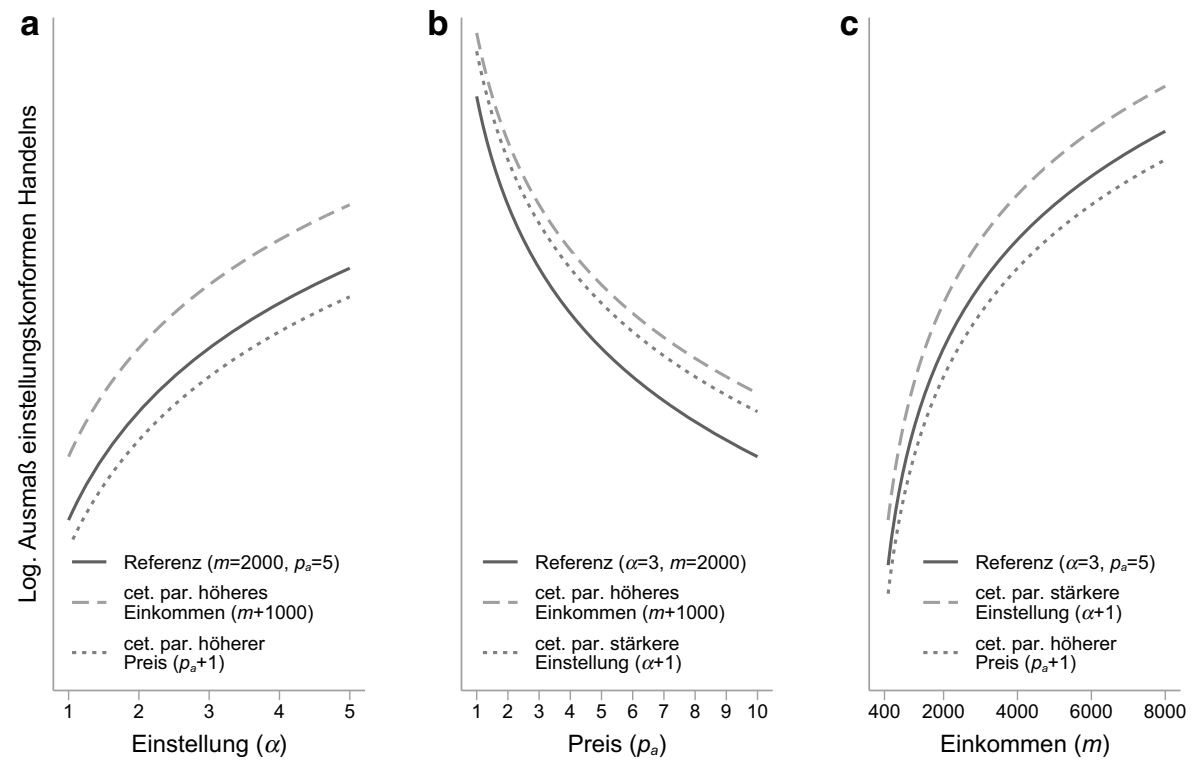

Abb. 1 Schematische Darstellung der anhand der Low-Cost-Hypothese zu erwartenden Zusammenhänge; dargestellt ist das vorhergesagte logarithmierte $A u s m a ß$ einstellungskonformen Verhaltens über die Einstellung ( $\alpha$; in Skalenpunkten; a), den Preis $\left(p_{a}\right.$; in $\left.€ ; \mathbf{b}\right)$ und das Einkommen $(m ;$ in $€ ; \mathbf{c})$

ein negativer Effekt der Kosten einstellungskonformen Handelns sowie die kostenabhängige Stärke des Einstellungseffekts. ${ }^{5}$ Neben der theoretischen Fundierung der im Rahmen der LCH erwarteten Zusammenhänge erlaubt das Modell zudem präzise Prognosen, da der funktionale Zusammenhang zwischen den relevanten Variablen klar spezifiziert ist.

Durch Logarithmieren der Nachfragefunktion erhält man schließlich die Gleichung

$$
\ln x_{a}^{*}\left(\alpha, m, p_{a}\right)=\ln \alpha+\ln m-\ln p_{a},
$$

mithilfe derer die postulierten Zusammenhänge in Abb. 1 grafisch veranschaulicht werden. Das anhand des Modells zu erwartende Ausmaß einstellungskonformen Handelns ist jeweils schematisch über die Stärke der Einstellung (Abb. 1a), die Höhe des Preises einstellungskonformen Handelns (Abb. 1b) und die Höhe des verfügbaren Einkommens (Abb. 1c) abgetragen (durchgezogene Linie). Die gepunktete und die gestrichelte Linie stellen dar, wie sich der Zusammenhang verschiebt, wenn die jeweilige Einflussgröße unter Konstanthalten der anderen Variablen erhöht wird. So wird etwa deutlich, dass der Anstieg des Ausmaßes einstellungskonformen Handelns mit zunehmender Stärke der Einstellung abnimmt, was der Vorstellung eines abnehmenden Grenznutzens entspricht. Wird lediglich der Preis einstellungskonfor-

\footnotetext{
${ }_{5}^{5}$ Die vermuteten Zusammenhänge gehen aus entsprechenden partiellen Ableitungen hervor (siehe Übersicht in Tab. 4 im Anhang).
} 
men Handelns erhöht, verschiebt sich die Linie nach unten - das jedoch stärker bei stärker ausgeprägter Einstellung, da hier auf deutlich höherem Niveau einstellungskonformes Handeln „nachgefragt“ wird. Wird statt des Preises ausschließlich das verfügbare Einkommen erhöht, verschiebt sich die Kurve entsprechend nach oben.

\subsection{Bisherige Prüfung der neuen Modellierung}

Tutić et al. (2017) illustrieren die verbesserte Teststrategie anhand einer Reanalyse der Datengrundlage zweier bereits publizierter Studien, einerseits zur monetären Bewertung der Biodiversität in Wäldern (Liebe 2007) und andererseits zu Einstellungen gegenüber Tieren (Liebe und Jahnke 2017). In beiden Fällen scheitert die LCH unter Verwendung der vormals üblichen Teststrategie, welche die Aufnahme eines expliziten Interaktionsterms zwischen der Einstellung (Umweltbewusstsein bzw. Bewusstsein für Tierwohl) und der Kostspieligkeit einstellungskonformen Verhaltens (geringeres Einkommen) fordert. Anhand der neuen (theoretisch fundierten) Teststrategie finden Tutić et al. (2017) hingegen durchaus Unterstützung für die LCH.

Es muss jedoch eingewandt werden, dass die Reanalyse bereits publizierten Materials gerade zum Test einer verbesserten Teststrategie und vor dem Hintergrund der teils widersprüchlichen Befundlage (s. Abschn. 1.1) zwar sinnvoll scheint, damit aber auch Beschränkungen der Datengrundlage in Kauf genommen werden müssen. So wird in den genannten Anwendungsfällen die Zahlungsbereitschaft für Biodiversität im Wald oder Spenden an eine Tierschutzorganisation als Ausmaß einstellungskonformen Handelns betrachtet. Die Kosten einstellungskonformen Handelns werden sodann über das Haushaltsäquivalenzeinkommen operationalisiert. Dies stellt in zweierlei Hinsicht ein Problem dar. Zum einen ist die lediglich über das Haushaltsäquivalenzeinkommen erfasste Kostspieligkeit einstellungskonformen Handelns (ohne eine exogen variierte Kostenkomponente in Form eines Preises einstellungskonformen Handelns) endogen, was potenziell zu verzerrten Ergebnissen führen kann. Zum anderen ermöglicht dieses Vorgehen lediglich einen Test der LCH im weiteren Sinne eines beschränkten Einkommens (die Autoren sprechen in diesem Zusammenhang selbst auch von einer Low-Income-Hypothese [LIH]), nicht aber der LCH im engeren Sinne eines Preises einstellungskonformen Handelns. Nach bestem Wissen existiert eine solche Prüfung der LCH (im engeren Sinn) anhand der von Tutić et al. (2017) vorgeschlagenen Teststrategie bisher nicht. Die vorliegende Studie soll am Anwendungsfall der Bereitschaft, eine City-Maut zur Verbesserung der Luftqualität zu befürworten, einen ersten Test der LCH anhand der verbesserten Teststrategie liefern.

\subsection{Das Anwendungsbeispiel einer City-Maut}

Das Modell lässt sich unter anderem auf den vorliegenden Anwendungsfall der Befürwortung einer City-Maut zur Verbesserung der Luftqualität übertragen. So kann etwa vermutet werden, dass mit einem hohen Umweltbewusstsein auch eine erhöhte Bereitschaft zu umweltbewussten Verhaltensweisen einhergeht. Es wird angenommen, dass der intrinsische Nutzen aus umweltbewussten Verhaltensweisen umso 
größer ist, je ausgeprägter das Umweltbewusstsein ist. Im Fall von Umweltschäden, wie sie unter anderem durch die straßenverkehrsbedingten Emissionen verursacht werden, müsste sich bei ausgeprägtem Umweltbewusstsein ein hoher intrinsischer Nutzen aus Verhaltensweisen, die zur Beseitigung der Umweltschäden beitragen, ergeben. Davon ausgehend, dass eine City-Maut zu einer Verringerung der Emissionen und damit zur Verbesserung der (innerstädtischen) Luftqualität beiträgt, wird also ein positiver Effekt des Umweltbewusstseins auf die Absicht, eine City-Maut zu befürworten, erwartet (vgl. Abb. 1a).

Da Akteure allerdings nur über begrenzte Ressourcen verfügen, kann eine mit steigender Kostspieligkeit abnehmende Bereitschaft zur Befürwortung einer City-Maut erwartet werden. Auch Personen mit ausgeprägtem Umweltbewusstsein verfügen nur über begrenzte Ressourcen, können mithin ihre Präferenzen nicht unmittelbar umsetzen, sondern nur soweit, wie es die Rahmenbedingungen zulassen. Somit kann schließlich ein mit steigender Kostspieligkeit abnehmender Einfluss des Umweltbewusstseins auf die Befürwortung der Maut erwartet werden. Dabei spielt einerseits die Höhe der Mautgebühren im Sinne eines Preiseffekts eine Rolle (vgl. Abb. 1b), andererseits aber auch die Ressourcenausstattung der Akteure im Sinne eines Einkommenseffekts (vgl. Abb. 1c). Zusammenfassend lässt sich festhalten: Je höher die Mautgebühren sind, aber auch je geringer das zur Verfügung stehende Einkommen ist, desto geringer ist der Einfluss des Umweltbewusstseins. Im vorliegenden Beitrag wird untersucht, ob sich diese Zusammenhänge in der erwarteten Weise zeigen (sie also den im theoretischen Modell formal dargestellten Beziehungen folgen; s. auch Abschn. 3.2).

Basierend auf diesen Ausführungen lässt sich zudem argumentieren, dass die vermuteten Zusammenhänge insbesondere für die Subgruppe an Personen, die in ihrem Mobilitätsverhalten direkt von einer möglichen Maut betroffen wären, bestehen sollten. So dürfte die Höhe der Mautgebühren etwa gerade für Personen, die (häufig) mit einem Kraftfahrzeug in einer der möglichen Mautzonen fahren, entscheidungsrelevant sein. Diese hätten bei Einführung einer Maut individuelle Kosten zu tragen, während sich die Einführung einer Maut für andere Subgruppen (etwa Personen, die derzeit über kein selbstgenutztes Kraftfahrzeug verfügen) primär in veränderten Rahmenbedingungen der künftigen Verkehrsmittelwahl niederschlüge. In den empirischen Analysen wird weiterhin geprüft, ob die Ergebnisse für unterschiedliche Messungen der Kostenkomponente substanziell robust bleiben. Abschließend werden die mithilfe der verbesserten Modellierung gewonnenen Ergebnisse solchen gegenübergestellt, die auf der herkömmlichen Teststrategie basieren.

\section{Daten und Methoden}

Die nachfolgenden Analysen basieren auf Daten einer im Frühsommer 2018 postalisch durchgeführten Bevölkerungsbefragung. Die Befragung wurde begleitend zur Methodenausbildung am Institut für Soziologie der Ludwig-Maximilians-Universität München durchgeführt. Hierzu wurde aus den jeweiligen Melderegistern eine Zufallsstichprobe der Wohnbevölkerung Münchens sowie einiger Umlandgemeinden mit hoher Pendelverflechtung zu München gezogen. Für München wurden 3400 Per- 
sonen gezogen, für die Umlandgemeinden jeweils 500 aus Gröbenzell, Landshut, Poing und Rosenheim. Insgesamt wurden 1335 der 5400 verschickten Fragebögen ausgefüllt zurückgesandt. Abzüglich 162 unzustellbarer Fragebögen wird somit eine (bereinigte) Ausschöpfungsquote von 26\% realisiert. Aufgrund teilweise fehlender Angaben beschränkt sich das Analysesample auf 4317 Vignettenurteile von 1102 Personen.

\subsection{Operationalisierung}

Im Rahmenfragebogen wurde Umweltbewusstsein anhand der von Diekmann und Preisendörfer (1998) entwickelten Skala zur Erfassung des allgemeinen Umweltbewusstseins erhoben. Damit wird die in der Literatur gängige Messung aufgegriffen und so die Vergleichbarkeit von Ergebnissen ermöglicht. Die Skala umfasst neun Items, die sich in eine affektive, eine kognitive und eine konative Komponente einteilen lassen. Die affektive Komponente bezieht sich auf emotionale Betroffenheit durch Umweltprobleme, die kognitive Komponente beschreibt die Einsicht, dass ein (durch Menschen verursachtes) Umweltproblem besteht und die konative Komponente zielt auf die grundsätzliche Bereitschaft zu individuellen oder kollektiven Handlungen zur Bekämpfung des Problems. Alle Items werden auf einer fünfstufigen Rating-Skala erfasst. Basierend auf einer Faktorenanalyse ergibt sich unter Einbezug aller neun Items eine eindimensionale Messung des Umweltbewusstseins, bei der $38 \%$ der Varianz der Items erklärt werden und alle Faktorladungen über 0,4 liegen. Die neun Items werden also zu einem additiven Index zusammengefasst (Cronbachs $\alpha=0,85){ }^{6}$ Dieser weist einen Mittelwert von 3,77 (mit einem Minimum von 1, einem Maximum von 5 und einer Standardabweichung von 0,75) auf.

Die Befürwortung einer möglichen City-Maut in München wurde mithilfe eines faktoriellen Surveys erhoben. Dieses Vorgehen erlaubt, die Vorteile eines experimentellen Designs im Rahmen einer Befragung zu nutzen (s. bspw. Auspurg und Hinz 2015). Den Befragten wurden jeweils vier hypothetische Mautmodelle (Vignetten) zur Bewertung vorgelegt, deren Merkmale (Dimensionen) sich in der jeweiligen Ausgestaltung unterscheiden. Die variierten Dimensionen umfassen neben der Höhe der Mautgebühren u. a. das zu erwartende Ausmaß der Luftverbesserung oder den Geltungsbereich der Maut. Es wurde, den methodischen Empfehlungen zur Erstellung faktorieller Surveys folgend, eine effiziente Auswahl der verwendeten 1200 unterschiedlichen Vignetten getroffen. Dabei werden die Vignetten so gewählt, dass

\footnotetext{
${ }^{6}$ Die Items umfassen die folgenden Aussagen: (1) „Es beunruhigt mich wenn ich daran denke, unter welchen Umweltverhältnissen unsere Kinder und Enkelkinder wahrscheinlich leben müssen.“ (2) ,Wenn wir so weitermachen wie bisher, steuern wir auf eine Umweltkatastrophe zu.“ (3) „Wenn ich Zeitungsberichte über Umweltprobleme lese oder entsprechende Fernsehsendungen sehe, bin ich oft empört und wütend." (4) „Es gibt Grenzen des Wachstums, die unsere industrialisierte Welt schon überschritten hat oder sehr bald erreichen wird.“(5) „Derzeit ist es immer noch so, dass sich der größte Teil der Bevölkerung wenig umweltbewusst verhält.“(6) „Nach meiner Einschätzung wird das Umweltproblem in seiner Bedeutung von vielen Umweltschützern stark übertrieben.“ (7) „Es ist immer noch so, dass die Politiker/innen viel zu wenig für den Umweltschutz tun.“ (8), Zugunsten der Umwelt sollten wir alle bereit sein, unseren derzeitigen Lebensstandard zu senken.“ (9) „Umweltschutzmaßnahmen sollten auch dann durchgesetzt werden, wenn dadurch Arbeitsplätze verloren gehen.“.
} 
Nach Einschätzung von Experten würde die City-Maut die Luftqualität im Stadtgebiet München um 20\% verbessern, wobei an übermäßig belasteten Kreuzungen eine besonders starke Luftverbesserung zu erwarten wäre. Das Verkehrsaufkommen im Stadtgebiet wird voraussichtlich etwas geringer werden.

Die Mautgebühr beträgt im gesamten Stadtgebiet (ohne Autobahn) für alle Fahrzeuge $10 €$ pro Tag.

Für Anwohner in Mautzonen ist keine Mautbefreiung vorgesehen. Die Mauteinnahmen werden für Baumaßnahmen zur Stauvermeidung verwendet.

Sollte ein solches Maut-Modell Ihrer Meinung nach eingeführt werden?

\begin{tabular}{|c|c|c|c|c|c|c|c|c|c|c|}
\hline $\begin{array}{c}\text { Auf keinen } \\
\text { Fall }\end{array}$ & & & & & $\begin{array}{l}\text { Unent- } \\
\text { schlossen }\end{array}$ & & & & & $\begin{array}{c}\text { Auf jeden } \\
\text { Fall }\end{array}$ \\
\hline-5 & -4 & -3 & -2 & -1 & 0 & +1 & +2 & +3 & +4 & +5 \\
\hline 0 & 0 & 0 & 0 & 0 & 0 & 0 & 0 & 0 & 0 & 0 \\
\hline
\end{tabular}

Abb. 2 Beispielvignette mit Antwortskala (variierte Dimensionen unterstrichen)

bei möglichst geringen Korrelationen zwischen den Dimensionen maximale Varianz der Level vorliegt. So wird die Präzision der geschätzten Effekte der Vignettendimensionen auf das Vignettenurteil erhöht (bspw. Auspurg und Hinz 2015). ${ }^{7}$ Durch die randomisierte Zuweisung der Vignetten (und damit der experimentellen Stimuli) ist zudem sichergestellt, dass die Vignettendimensionen nicht mit Merkmalen der Befragten korrelieren.

Abbildung 2 zeigt eine Beispielvignette sowie die elfstufige Rating-Skala, inwiefern das beschriebene Mautmodell eingeführt werden sollte $(-5=$,, auf keinen Fall“" bis 5=,,auf jeden Fall“). Um in der weiteren Analyse das Vignettenurteil in logarithmierter Form verwenden zu können, wird es auf einen Wertebereich von 1-11 reskaliert, wobei hohe Werte weiterhin positivere Bewertungen widerspiegeln. Der Grad der zum jeweiligen Mautmodell geäußerten Zustimmung wird als einstellungskonforme Verhaltensabsicht, das Mautmodell auch im Rahmen einer (verbindlichen) Abstimmung zu befürworten, angesehen. Wie bereits angedeutet, handelt es sich dabei nicht um tatsächliches Verhalten. Mithin fallen auch die damit einhergehenden Kosten nicht unmittelbar an. Die Einführung einer City-Maut brächte allerdings durchaus umfassende Auswirkungen auf verhaltensrelevante Rahmenbedingungen mit sich. Es kann also argumentiert werden, dass die Absicht, ein solches Mautmodell zu befürworten, auch die Bereitschaft umfasst, tatsächlich anfallende (Verhaltens-)Kosten zu tragen und so als einstellungskonforme, mittelbar kostspielige Verhaltensabsicht betrachtet werden kann.

\footnotetext{
7 Technisch ausgedrückt wurde ein sogenanntes D-effizientes Design gewählt, bei dem auch alle Zweifach- und Dreifachinteraktionen zwischen den Dimensionen orthogonalisiert wurden. Für eine Übersicht der variierten Dimensionen siehe Tab. 5 im Anhang.
} 
Dieses Vorgehen scheint auch empirisch gerechtfertigt. Zwar existieren bisher nur wenige und zudem teils widersprüchliche Befunde zur Validität der in Survey-Experimenten gemessenen Verhaltensabsichten in Bezug auf tatsächliches Verhalten. So finden u.a. Hainmueller et al. (2015) kongruente Ergebnisse für Verhaltensabsicht und tatsächliches Verhalten, während etwa Findley et al. (2017) deutliche Unterschiede zwischen den Messungen berichten. Insgesamt scheint sich jedoch abzuzeichnen, dass bei Verhaltensweisen, die kaum mit sozialer Erwünschtheit besetzt sind, von einer hohen Übereinstimmung zwischen Verhaltensabsicht und tatsächlichem Verhalten (zumindest hinsichtlich der Richtung und relativen Stärke von Effekten) ausgegangen werden kann (Petzold und Wolbring 2019, S. 8). Im vorliegenden Fall kann zwar durchaus argumentiert werden, dass die Befürwortung geeigneter Maßnahmen zur (innerstädtischen) Luftverbesserung sozial erwünscht sei, nicht jedoch, welche Maßnahme (etwa eine City-Maut oder Dieselfahrverbote) die zu befürwortende ist.

Inwieweit die einstellungskonforme Bereitschaft zur Befürwortung der Maut als eine kostspielige Verhaltensabsicht angesehen werden kann, richtet sich sowohl nach dem zur Verfügung stehenden Einkommen, operationalisiert anhand des Haushaltsnettoäquivalenzeinkommens mit einem Mittelwert von 2518,78€ (einem Minimum von 400, einem Maximum von 20.000 und einer Standardabweichung von 1520,88), als auch nach der Höhe des Preises einstellungskonformen Handelns in Form der anfallenden Mautgebühren. Letztere wurden in Abstufungen zwischen 1 und $10 €$ pro Tag variiert. ${ }^{8}$

Im Rahmen weiterer Robustheitsanalyen wird zudem eine alternative Operationalisierung der Kosten herangezogen, die dem Umstand Rechnung trägt, dass die Kostspieligkeit einer City-Maut insbesondere für Personen, die in ihrem jeweiligen Mobilitätsverhalten direkt betroffen wären, entscheidungsrelevant sein sollte. Hierbei stehen folglich die bei gegebenem Mobilitätsverhalten tatsächlich anfallenden Mautgebühren im Fokus. Die Höhe der individuell zu erwartenden Kosten errechnet sich aus der Höhe der Mautgebühren, multipliziert mit der Häufigkeit, in der Befragte ein Kraftfahrzeug in einer der möglichen Mautzonen nutzen. ${ }^{9}$ Dieser Kostenterm wird für Personen, für deren Kraftfahrzeug eine entsprechende Maut nicht gelten soll oder die aufgrund einer Befreiung für Anwohner von der Zahlung von Mautgebühren ausgenommen wären, auf $0,01 €$ gesetzt. So können einerseits die Kosten einstellungskonformer Befürwortung auch weiterhin in logarithmierter Form in das Modell aufgenommen werden (s. Gleichung 3) und andererseits ist abgebildet, dass

\footnotetext{
8 Die Anzahl möglicher Ausprägungen (Level) der Höhe der Mautgebühren wurde zwischen zwei zufällig ausgewählten Teilsplits von jeweils $50 \%$ der Befragten variiert. Für einen Teil der Befragten variierte die Höhe zwischen 1, 5 und $10 €$ pro Tag, für den anderen Teil der Befragten wurden zudem weitere Abstufungen von $3 €$ und $7 €$ pro Tag aufgenommen, um einen möglichen nichtlinearen Effekt der Mautgebühren besser schätzen zu können. Im Folgenden sind die gepoolten Ergebnisse dargestellt. Die berichteten $\mathrm{Zu}-$ sammenhänge zeigen sich substanziell, aber auch für getrennte Analysen der beiden Teilsplits (mit viel bzw. wenig Variation der Höhe der Mautgebühren).

9 Die Häufigkeit der Autonutzung wird über die Angabe, an wie vielen Tagen Befragte in der vergangenen Woche mit einem Auto im jeweiligen Stadtgebiet gefahren sind („Gar nicht“, „An ein bis zwei Tagen“, „An drei bis vier Tagen“, „An fünf bis sechs Tagen“, „Täglich“), gemessen. Es wird jeweils der Mittelwert der Angabe (also etwa 1,5 Tage für „An ein bis zwei Tagen“) angesetzt.
} 
diesen Personen faktisch keine direkten Kosten in Form zu leistender Mautgebühren entstünden.

\subsection{Analysemodell}

Wie bereits erläutert, lässt sich anhand der logarithmierten Nachfragefunktion nach einstellungskonformem Handeln das funktionale Zusammenspiel der im Rahmen der LCH relevanten Variablen als Reihe linearer Beziehungen darstellen (s. Gleichung 2). Die postulierten Zusammenhänge sollen entsprechend anhand des linearen Regressionsmodells

$$
\ln x_{a}=\text { const }+\gamma_{\alpha} \ln \alpha+\gamma_{m} \ln m+\gamma_{p} \ln p_{a}+\varepsilon
$$

getestet werden. Ein strenger Test des Modells fordert, dass die Koeffizienten sich jeweils nicht signifikant von 1 (Umweltbewusstsein, Einkommen) oder -1 (Mautgebühren) unterscheiden (s. Gleichung 2). Wie auch Tutić et al. (2017) argumentieren, genügt in der Forschungspraxis ein moderaterer Test. So wird berücksichtigt, dass die Stärke der Einstellung entgegen der theoretischen Modellierung kein absolutes Skalenniveau aufweist. Zudem ist auf das Problem unbeobachteter Heterogenität hinzuweisen, das sich aus der der Nachfragefunktion nach einstellungskonformem Handeln inhärenten Annahme sonst gleicher Bedingungen ergibt. Während dem Problem unbeobachteter Heterogenität durch die randomisierte Zuweisung der Vignetten begegnet wird, kann insbesondere bei der vorliegenden Messung der Einstellung nicht von einem absoluten Skalenniveau ausgegangen werden. Es wird im Rahmen des moderaten Tests also lediglich geprüft, ob die Koeffizienten in die erwartete Richtung weisen, wobei $\gamma_{\alpha}>0, \gamma_{m}>0$ und $\gamma_{p}<0$ jeweils statistisch signifikant sein müssen.

\section{Ergebnisse}

Deskriptiv zeigt sich, dass der gesamte Wertebereich möglicher Mautbewertungen ausgeschöpft wird (s. Abb. 3). Mit einem Mittelwert von 5,39 (Standardabweichung von 3,59) werden die Mautmodelle insgesamt eher kritisch bewertet. Das zeigt sich auch an dem vergleichsweise hohen Anteil stark ablehnender Mautbewertungen. So werden etwa $27 \%$ der vorgelegten Mautmodelle vollständig abgelehnt (Vignettenurteile = 1). Zugleich werden aber auch $41 \%$ der Mautmodelle eher oder stark befürwortet (Vignettenurteile $>6$ ).

\subsection{Verbesserte Teststrategie zur Prüfung der $\mathrm{LCH}$}

Der Argumentation der LCH folgend sollte sich der negative Effekt der Höhe der Mautgebühren mit steigendem Umweltbewusstsein abschwächen. Um dies zu prüfen, wird das oben skizzierte Modell geschätzt (s. Gleichung 3), wobei die zentralen Variablen, wie ausgeführt, in logarithmierter Form eingehen. In Abb. 4 sind vorhergesagte Werte der logarithmierten Befürwortung einer Maut zunächst grafisch über 


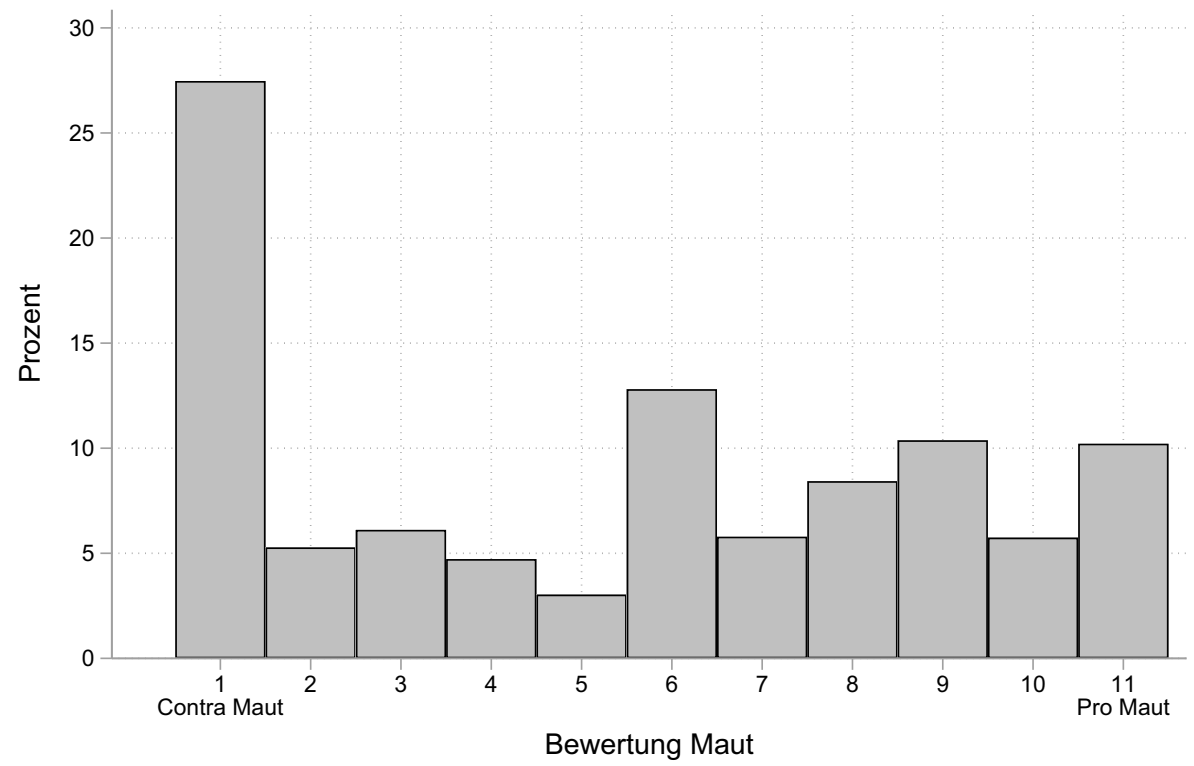

Abb. 3 Verteilung der Mautbewertungen (Darstellung basiert auf 4317 Vignettenurteilen von 1102 Personen)

das Umweltbewusstsein (Abb. 4a), die Höhe der Mautgebühren (Abb. 4b) und die Höhe des Haushaltsnettoäquivalenzeinkommens (Abb. 4c) dargestellt, wobei jeweils dieselben Referenzwerte wie in Abb. 1 eingesetzt sind.

Es zeigt sich das erwartete Muster eines negativen Effekts der Höhe der Mautgebühr und positiver Effekte des Einkommens sowie des Umweltbewusstseins, die jeweils statistisch signifikant $(p<0,05)$ sind (siehe Tab. 1). Der Koeffizient des Umweltbewusstseins unterscheidet sich entsprechend der Forderungen eines strengen Tests zudem nicht signifikant vom Wert 1 (Wald-Test, $\mathrm{F}(1,1101)=0,05, p=0,815$ ). Die übrigen Koeffizienten weisen zwar ebenfalls in die jeweils erwartete Richtung, unterscheiden sich jedoch von den anhand eines strengen Tests geforderten Werten von 1 für das Einkommen (Wald-Test, $\mathrm{F}(1,1101)=535,43, p<0,001$ ) und -1 für die Höhe der Mautgebühren (Wald-Test, $\mathrm{F}(1,1101)=3247,19, p<0,001$ ).

\subsection{Robustheitsanalysen}

Um die Ergebnisse abzusichern, werden verschiedene Robustheitsanalysen durchgeführt. In einem ersten Schritt wird die Struktur der Daten (es liegen mehrere Vignettenurteile pro Befragtem vor) den Empfehlungen zur Analyse faktorieller Surveys folgend durch die Verwendung eines Random-Intercept(RI)-Modells explizit berücksichtigt (Auspurg und Hinz 2015). Wie an den in Tab. 2 dargestellten Ergebnissen deutlich wird, weisen die Effekte erneut in die jeweils erwartete Richtung und sind statistisch signifikant $(p<0,05)$. Der Effekt des Umweltbewusstseins unterscheidet sich dabei zudem nicht signifikant von 1 (Wald-Test, $\chi^{2}(1)=0,05, p=0,831$ ), wohingegen sich die übrigen Koeffizienten von den im Rahmen eines strengen Tests ge- 
a

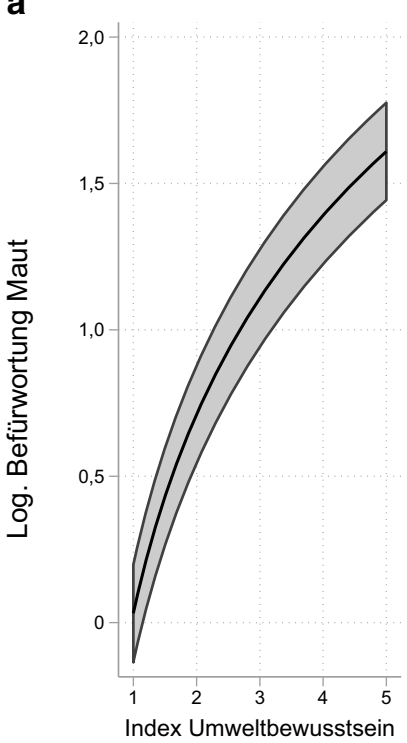

b

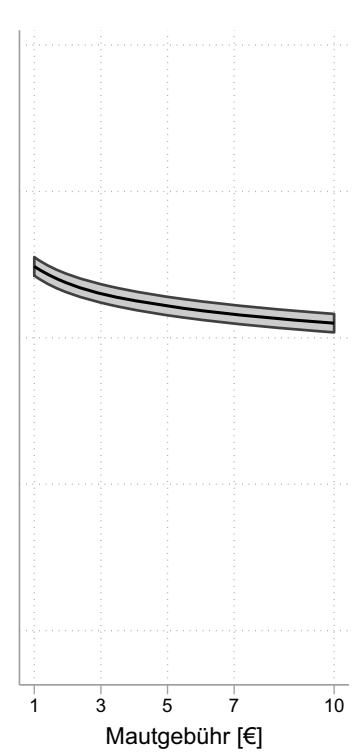

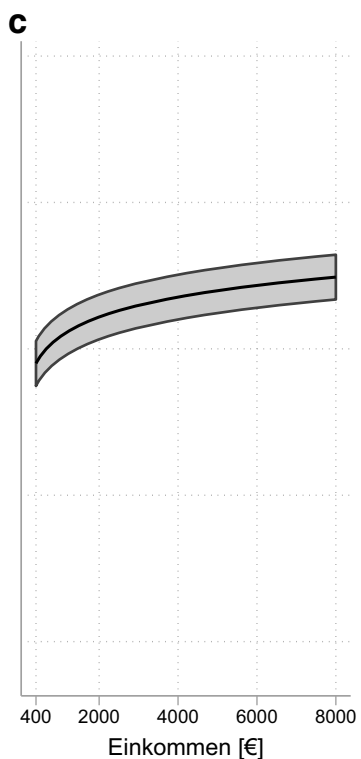

Abb. 4 Vorhergesagte Werte der logarithmierten Befürwortung einer Maut mit 95\%-Konfidenzintervallen, dargestellt über das Umweltbewusstsein (a; wobei $m=2000, p_{a}=5$ ), die Mautgebühr (b; wobei $\alpha=3, m=2000)$ und das Einkommen (c; wobei $\alpha=3, p_{a}=5$ )

forderten Werten von 1 für das Einkommen (Wald-Test, $\chi^{2}(1)=534,60, p<0,001$ ) und -1 für die Höhe der Mautgebühren (Wald-Test, $\chi^{2}(1)=3976,66, p<0,001$ ) unterscheiden. Insgesamt bleiben die berichteten Ergebnisse also auch unter Verwendung des RI-Modells robust. Darüber hinaus wird deutlich, dass mit rund $43 \%$ ein substanzieller Anteil der Varianz in den Vignettenurteilen auf Unterschiede zwischen den Befragten zurückführbar ist.

Vor diesem Hintergrund deutet die in Abb. 3 dargestellte Antwortverteilung darauf hin, dass es sich bei der Bewertung der Mautmodelle möglicherweise um einen zweiteiligen Entscheidungsprozess handelt, bei dem zunächst entschieden wird, ob eine Maut überhaupt in Erwägung gezogen wird und erst im zweiten Schritt (gegebenenfalls) eine Beurteilung des konkreten Modells vorgenommen wird. Sollte dies der Fall sein, könnten Schätzer einstufiger Regressionsverfahren verzerrt sein. Entsprechend wird hier zudem ein zweistufiges Vorgehen gewählt, das dem hohen Anteil stark negativer Bewertungen der Mautmodelle Rechnung trägt. Auf Stufe 1 wird ein Probitmodell geschätzt, wobei die abhängige Variable lediglich unterscheidet, ob eine Maut überhaupt in Erwägung gezogen wird (d.h. Abstufungen jenseits der Option ,auf keinen Fall“ vorgenommen werden). Feingliedrige Abstufungen der Bewertung des jeweiligen Mautmodells gehen sodann auf Stufe 2 in ein trunkiertes lineares Regressionsmodell ein. Ergebnisse dieses sogenannten Craggit-Modells (Cragg 1971), das die Schätzungen beider Stufen kombiniert, sind ebenfalls in Tab. 2 dargestellt. Burke (2009) folgend sind neben den getrennten Ergebnissen für beide Stufen auch durchschnittliche Marginaleffekte (AMEs) berechnet, die den Effekt der unabhängigen Variablen auf beiden Stufen zusammenfassen. Sie geben an, um 
Tab. 1 Lineares Regressionsmodell zur Prüfung der LowCost-Hypothese

\begin{tabular}{ll}
\hline & Log. Befürwortung Maut \\
\hline Log. Umweltbewusstsein & $0,980^{* * *}$ \\
& $(0,085)$ \\
Log. Einkommen (in $€)$ & $0,098^{*}$ \\
& $(0,039)$ \\
Log. Mautgebühr (in $€)$ & $-0,084^{* * *}$ \\
& $(0,016)$ \\
Konstante & $-0,580^{+}$ \\
& $(0,321)$ \\
Adj. $R^{2}$ & 0,066 \\
$N$ (Vignetten) & 4317 \\
$N$ (Personen) & 1102 \\
\hline
\end{tabular}

Koeffizienten und cluster-robuste Standardfehler auf Befragtenebene in Klammern, um die Datenstruktur (mehrere Vignettenurteile pro Befragtem) zu berücksichtigen

$+p<0,10 ;{ }^{*} p<0,05 ;{ }^{* *} p<0,01 ;{ }^{* * *} p<0,001$

Tab. 2 Robustheitsanalysen zur Absicherung der Befunde zur Low-Cost-Hypothese anhand verschiedener Regressionsmodelle der logarithmierten Befürwortung einer City-Maut

\begin{tabular}{|c|c|c|c|c|c|}
\hline & \multirow[t]{2}{*}{$\mathrm{RI}$} & \multicolumn{3}{|l|}{ Craggit $^{\mathrm{a}}$} & \multirow[t]{2}{*}{ OLS } \\
\hline & & Stufe 1 & Stufe 2 & AMEs & \\
\hline Log. Umweltbewusstsein & $\begin{array}{l}0,982^{* * * *} \\
(0,085)\end{array}$ & $\begin{array}{l}1,334^{* * * *} \\
(0,140)\end{array}$ & $\begin{array}{l}0,260^{* * * *} \\
(0,060)\end{array}$ & $\begin{array}{l}0,960^{* * *} \\
(0,059)\end{array}$ & $\begin{array}{l}0,939^{* * *} \\
(0,084)\end{array}$ \\
\hline Log. Einkommen (in $€$ ) & $\begin{array}{c}0,098^{*} \\
(0,039)\end{array}$ & $\begin{array}{c}0,131^{*} \\
(0,062)\end{array}$ & $\begin{array}{r}0,030 \\
(0,022)\end{array}$ & $\begin{array}{l}0,098^{* * *} \\
(0,022)\end{array}$ & $\begin{array}{l}0,118^{* *} \\
(0,039)\end{array}$ \\
\hline Log. Mautgebühr (in $€$ ) & $\begin{array}{l}-0,100^{* * *} \\
(0,014)\end{array}$ & $\begin{array}{l}-0,095^{\text {*** }} \\
(0,025)\end{array}$ & $\begin{array}{l}-0,040^{* * *} \\
(0,010)\end{array}$ & $\begin{array}{l}-0,084^{* * *} \\
(0,014)\end{array}$ & - \\
\hline $\begin{array}{l}\text { Log. zu erwartende Kosten } \\
\text { (in } € \text { ) }\end{array}$ & - & - & - & - & $\begin{array}{l}-0,041^{* * *} \\
(0,007)\end{array}$ \\
\hline Konstante & $\begin{array}{l}-0,553^{+} \\
(0,322)\end{array}$ & $\begin{array}{l}-2,000^{* * *} \\
(0,518)\end{array}$ & $\begin{array}{l}1,320^{* * *} \\
(0,177)\end{array}$ & - & $\begin{array}{l}-0,934^{* *} \\
(0,322)\end{array}$ \\
\hline$\sigma^{\mathrm{b}}$ & - & $\begin{array}{l}0,491^{* * *} \\
(0,008)\end{array}$ & - & - & - \\
\hline$\rho^{\mathrm{c}}$ & 0,426 & - & - & - & - \\
\hline Adj. $R^{2}$ & 0,066 & - & - & - & 0,074 \\
\hline$N$ (Vignetten) & 4317 & 43 & & - & 4317 \\
\hline$N$ (Personen $)$ & 1102 & 11 & & - & 1102 \\
\hline
\end{tabular}

Koeffizienten und cluster-robuste Standardfehler auf Befragtenebene in Klammern, um die Datenstruktur (mehrere Vignettenurteile pro Befragtem) zu berücksichtigen. Berichtet sind die Ergebnisse eines Random-Intercept-Modells, eines Craggit-Modells (Cragg 1971) sowie eines linearen Regressionsmodells mit einer alternativen Kostenmessung

${ }^{\mathrm{a}}$ Für Stufe 1 sind Koeffizienten eines Probitmodells, ob überhaupt ein Mautmodell in Erwägung gezogen wird ( $y>1$ vs. $y=1$ ), dargestellt. Für Stufe 2 sind die Koeffizienten eines trunkierten linearen Regressionsmodells der Bewertung der Mautmodelle $(y>1)$ dargestellt. Die berichteten Average Marginal Effects (AMEs) fassen beide Stufen zusammen. Die Standardfehler der AMEs sind mithilfe des von Burke (2009) vorgeschlagenen Bootstrap-Verfahrens unter Verwendung des Stata ados craggit mit jeweils 100 Iterationen berechnet

$\mathrm{b}_{\sigma}$ gibt die geschätzte Fehlervarianz des Probitmodells an

${ }^{\mathrm{c}} \rho$ gibt die Intraclusterkorrelation des Random-Intercept-Modells an

$+p<0,10 ;^{*} p<0,05$; $^{* *} p<0,01 ;{ }^{* * *} p<0,001$ 
wie viele Skalenpunkte ein Mautmodell besser bewertet wird, wenn sich die unabhängige Variable um eine Einheit erhöht. Entsprechende Standardfehler für die AMEs werden anhand des von Burke (2009) vorgeschlagenen Bootstrap-Verfahrens geschätzt.

Es zeigt sich, dass die Ergebnisse substanziell robust bleiben. Sowohl bei der Frage, ob eine Maut überhaupt in Erwägung gezogen wird (Stufe 1), als auch bei der Frage, in welchem Ausmaß ein solches Mautmodell befürwortet wird (Stufe 2), weisen die Effekte in die erwartete Richtung. Selbiges gilt für die durchschnittlichen Marginaleffekte, die beide Stufen zusammenfassen. Die AMEs sind jeweils statistisch signifikant ( $p<0,001)$, unterscheiden sich jedoch auch signifikant von den im Rahmen eines strengen Tests geforderten Werten von -1 für die Höhe der Mautgebühren (Wald-Test, $\chi^{2}(1)=3332,76, p<0,001$ ) und 1 für die Höhe des Einkommens (Wald-Test, $\left.\chi^{2}(1)=1284,65, p<0,001\right)$. Lediglich der durchschnittliche Marginaleffekt der Stärke des Umweltbewusstseins unterscheidet sich nicht signifikant von 1 (Wald-Test, $\chi^{2}(1)=0,46, p=0,497$ ), wie es ein strenger Test erfordert. Dennoch erfüllen die Ergebnisse beider Modellierungen die Anforderungen eines moderaten Tests, wie er angesichts der bereits ausgeführten Beschränkungen in der Forschungspraxis angemessen scheint.

In einem dritten Schritt soll geprüft werden, inwiefern die vermuteten Zusammenhänge im Speziellen für Personen bestehen, die in ihrem jeweiligen Mobilitätsverhalten direkt betroffen wären. Hierzu wird eine alternative Operationalisierung der individuellen Kosten einer City-Maut verwendet, die auf die tatsächlich zu erwartende Höhe anfallender Mautgebühren bei gegebenem Mobilitätsverhalten abzielt (s. Abschn. 3.1). Wie aus Tab. 2 ersichtlich ist, bleibt der Kernbefund auch unter Verwendung dieser alternativen Operationalisierung der Kosten einstellungskonformer Befürwortung bestehen. ${ }^{10}$

\subsection{Prüfung der LCH anhand der vormals üblichen Teststrategie}

Schließlich wird untersucht, inwiefern die vormals gängige Teststrategie, die LCH mithilfe eines Interaktionsterms aus Einstellung und Kosten zu prüfen, zu substanziell vergleichbaren Ergebnissen führt. Hierzu wird neben den Haupteffekten des Umweltbewusstseins und der Höhe der Mautgebühren ein multiplikativer Term in das Modell aufgenommen (s. Tab. 3). Neben der verbreiteten OLS-Modellierung wird auch die bereits angesprochene RI-Modellierung verwendet, um die genestete Struktur der Daten explizit zu berücksichtigen. Zur besseren Vergleichbarkeit ist wie auch in den vorigen Abschnitten die abhängige Variable der Befürwortung einer Maut jeweils logarithmiert, wobei die dargestellten Ergebnisse auch unter Verwendung der nichtlogarithmierten Form robust bleiben. Erwartungsgemäß zeigt sich in allen vier Modellen ein positiver Effekt des Umweltbewusstseins $(p<0,05)$ und ein negativer Effekt der Höhe der Mautgebühren $(p<0,05)$. Der im Rahmen der LCH zentrale Interaktionseffekt aus Einstellung und den Kosten einstellungskonfor-

\footnotetext{
10 Dieser Befund bleibt (für beide Kostenmessungen) auch dann bestehen, wenn im Rahmen weiterer Robustheitsanalysen sehr hohe Einkommen (über $8000 €$ Haushaltsnettoäquivalenzeinkommen) ausgeschlossen werden.
} 
Tab. 3 Lineare Regressionsmodelle der logarithmierten Befürwortung einer City-Maut zur Prüfung der Low-Cost-Hypothese anhand der vormals üblichen Teststrategie

\begin{tabular}{|c|c|c|c|c|}
\hline & \multicolumn{2}{|l|}{ OLS } & \multicolumn{2}{|l|}{ RI } \\
\hline & Ia & IIa & $\mathrm{Ib}$ & $\mathrm{IIb}$ \\
\hline Umweltbewusstsein & $\begin{array}{l}0,233^{* * *} \\
(0,042)\end{array}$ & $\begin{array}{l}0,267^{* * *} \\
(0,029)\end{array}$ & $\begin{array}{l}0,203^{* * *} \\
(0,039)\end{array}$ & $\begin{array}{l}0,270^{* * * *} \\
(0,029)\end{array}$ \\
\hline Mautgebühr (in $€$ ) & $\begin{array}{l}-0,058^{*} \\
(0,023)\end{array}$ & - & $\begin{array}{l}-0,084^{* * *} \\
(0,019)\end{array}$ & - \\
\hline Umweltbewusstsein $\times$ Mautgebühr & $\begin{array}{l}0,009 \\
(0,006)\end{array}$ & - & $\begin{array}{l}0,015^{* *} \\
(0,005)\end{array}$ & - \\
\hline Zu erwartende Kosten (in $€$ ) & - & $\begin{array}{l}-0,015^{* *} \\
(0,006)\end{array}$ & - & $\begin{array}{l}-0,013^{*} \\
(0,005)\end{array}$ \\
\hline $\begin{array}{l}\text { Umweltbewusstsein } \times \text { zu erwarten- } \\
\text { de Kosten }\end{array}$ & - & $\begin{array}{l}0,002 \\
(0,002)\end{array}$ & - & $\begin{array}{l}0,001 \\
(0,001)\end{array}$ \\
\hline Konstante & $\begin{array}{l}0,581^{* * *} \\
(0,165)\end{array}$ & $\begin{array}{l}0,364^{* *} \\
(0,114)\end{array}$ & $\begin{array}{l}0,725^{* * *} \\
(0,152)\end{array}$ & $\begin{array}{l}0,366^{* *} \\
(0,112)\end{array}$ \\
\hline$\rho^{\mathrm{a}}$ & - & - & 0,433 & 0,433 \\
\hline Adj. $R^{2}$ & 0,058 & 0,062 & 0,058 & 0,061 \\
\hline$N$ (Vignetten) & 1102 & 1102 & 1102 & 1102 \\
\hline$N$ (Personen) & 4317 & 4317 & 4317 & 4317 \\
\hline
\end{tabular}

Koeffizienten und cluster-robuste Standardfehler auf Befragtenebene in Klammern, um die Datenstruktur (mehrere Vignettenurteile pro Befragtem) zu berücksichtigen. Berichtet sind Ergebnisse für die Operationalisierung der Kosten anhand der Mautgebühr (Ia und Ib) bzw. der alternativen Messung anhand der zu erwartenden Kosten (IIa und IIb) jeweils unter Verwendung eines linearen Regressionsmodells bzw. eines Random-Intercept-Modells. Die abhängige Variable ist zur besseren Vergleichbarkeit mit den in Abschnitt 4.1 und 4.2 berichteten Modellen jeweils logarithmiert. Die dargestellten Effekte bleiben substanziell aber auch unter Verwendung der nichtlogarithmierten Form und unter Ausschluss sehr hoher Einkommen (über $8000 €$ Haushaltsnettoäquivalenzeinkommen) erhalten

${ }^{\mathrm{a}} \rho$ gibt die Intraclusterkorrelation des Random-Intercept-Modells an

$+p<0,10 ;{ }^{*} p<0,05 ;{ }^{* *} p<0,01 ;{ }^{* * *} p<0,001$

men Handelns kann jedoch nicht beobachtet werden. Zwar ist der Interaktionseffekt aus Umweltbewusstsein und Höhe der Mautgebühr im RI-Modell statistisch signifikant $(p<0,05)$, weist jedoch entgegen der theoretischen Erwartung kein negatives Vorzeichen auf.

Während die LCH anhand der vormals gängigen Teststrategie verworfen würde, liefert die verbesserte Teststrategie durchweg Unterstützung für die LCH. Die verbesserte Teststrategie scheint, zumindest basierend auf den vorliegenden Ergebnissen, eine (minimal) höhere Erklärungskraft aufzuweisen und zudem robuster gegenüber einzelnen Modellierungsentscheidungen (wie der konkreten Operationalisierung der Kosten einstellungskonformen Verhaltens) zu sein.

\section{Zusammenfassung}

Die vorliegende Arbeit zielte anhand der Befürwortung einer City-Maut in München auf einen Test der Implikationen, die sich aus der von Tutić et al. (2017) vorgeschlagenen neuerlichen Herleitung der LCH aus einem mikroökonomischen Modell ergeben. Dabei sollte in zweierlei Hinsicht an die umfassende Literatur um die LCH 
angeknüpft werden. Erstens sollte die LCH im engeren Sinn eines Preiseffekts, wie sie sich aus der verbesserten Teststrategie ergibt, einem ersten empirischen Test unterzogen und so die Ausführungen von Tutić et al. (2017) um einen zentralen Aspekt ergänzt werden. Zweitens sollte anhand des gewählten Anwendungsfalls untersucht werden, inwiefern bereits bei der vorgelagerten Entscheidung über die Gestaltung gesetzlicher Rahmenbedingungen trotz individuell anfallender Kosten Bereitschaft zu einstellungskonformer Befürwortung einer City-Maut besteht.

Den vorliegenden Ansatz zeichnet dabei aus, dass das experimentelle Design eines faktoriellen Surveys im Rahmen einer Bevölkerungsbefragung für den empirischen Test der LCH herangezogen wurde. Über 1300 Befragte aus München und einigen Umlandgemeinden mit hoher Pendelverflechtung zu München bewerteten insgesamt rund 5000 fiktive Mautmodelle. Aufgrund der randomisierten $\mathrm{Zu}-$ weisung der verschiedenen Mautmodelle (und damit der experimentellen Stimuli) konnte ausgeschlossen werden, dass Charakteristika der vorgelegten Mautmodelle mit soziodemografischen Merkmalen der Befragten korrelieren. So war es einerseits möglich, Bevölkerungsgruppen einzubeziehen, die in unterschiedlichem Maße von (den Kosten) einer möglichen City-Maut betroffen wären und so die sich aus der LCH ergebenden Implikationen anhand einer umfassenden Datengrundlage zu testen. Andererseits konnte die Höhe der Mautgebühren und damit die Kostspieligkeit einer möglichen City-Maut tatsächlich exogen variiert werden.

Empirisch zeigte sich eine breite Streuung der Bewertungen über alle vorgelegten Mautmodelle hinweg. Bezogen auf die LCH fanden sich durchaus Hinweise, die den erwarteten Zusammenhängen entsprechen. Während ein stärker ausgeprägtes Umweltbewusstsein sowie ein höheres Einkommen zu positiveren Bewertungen der Mautmodelle führten, sank die Zustimmung mit steigenden Mautgebühren. Die Effekte wiesen also in die erwartete Richtung und waren zudem statistisch signifikant. Im Rahmen weitergehender Analysen (Modellierung mithilfe eines RandomIntercept-Modells sowie eines Craggit-Modells, Ausschluss von Befragten mit sehr hohem Einkommen, Verwendung einer alternativen Operationalisierung der Kosten) erwies sich dieser Befund als sehr robust.

Abschließend wurden diese Ergebnisse analog zum Vorgehen von Tutić et al. (2017) mit auf der vormals gängigen Teststrategie basierenden Berechnungen verglichen. Letztere lieferten insgesamt keine Unterstützung für die LCH. Der im Fokus stehende Interaktionseffekt aus Einstellungs- und Kostenvariable wies entgegen der theoretischen Erwartung kein negatives Vorzeichen auf und reagierte in Stärke und Signifikanz auf einzelne Modellierungsentscheidungen. Neben der umfassenderen theoretischen Fundierung scheint die verbesserte Teststrategie also auch zu empirisch zuverlässigeren Resultaten zu führen, die zudem eine (wenngleich nur minimal) höhere Erklärungskraft aufwiesen.

\section{Diskussion}

Die vorliegende Analyse liefert einen wichtigen Beitrag zur Literatur um die LCH, indem die von Tutić et al. (2017) vorgeschlagene neuerliche Herleitung der LCH (im engeren Sinn eines Preiseffekts) einem ersten empirischen Test unterzogen wurde. 
Dazu wurden die Vorteile eines faktoriellen Surveys im Rahmen einer Bevölkerungsbefragung genutzt. Dieses experimentelle Design erlaubt die Prüfung der theoretisch vermuteten kausalen Zusammenhänge, die sich aus der LCH ergeben. Durch den breiten Einbezug verschiedener Aspekte einer möglichen City-Maut wurden den Befragten im Vergleich zu einfachen Itemabfragen komplexere, zugleich aber auch anschaulichere Stimuli vorgelegt (vgl. Auspurg und Liebe 2011).

Es wurde eine für faktorielle Surveys typische elfstufige Antwortskala verwendet. Dagegen kann eingewandt werden, dass dieses Vorgehen im Vergleich zu einem Choice-Experiment weiter von einer realitätsnahen Entscheidungssituation entfernt ist, wie sie etwa in einer möglichen Abstimmung über ein oder zwei konkrete Mautmodelle vorkommt (vgl. etwa Auspurg und Liebe 2011, S. 304). Allerdings mag die Möglichkeit feingliedriger Abstufungen des Vignettenurteils gerade in Situationen mit einer Vielzahl kritischer Bewertungen sinnvoll sein. Immerhin sind vorgenommene Abwägungen so auch dann beobachtbar, wenn Befragte dem vorgelegten Mautmodell insgesamt eher kritisch gegenüberstehen.

Die exogene Variation u. a. der Höhe der Mautgebühren stellt einen wesentlichen Vorteil des gewählten experimentellen Designs dar. Dennoch muss darauf hingewiesen werden, dass aus den exogen variierten Mautgebühren nur eingeschränkt auf die tatsächlich mit einer möglichen City-Maut einhergehenden Kosten geschlossen werden kann. Immerhin könnte durch den Umstieg auf andere Verkehrsmittel oder eine grundsätzliche Anpassung des eigenen Mobilitätsverhaltens (Vermeidung unnötiger Wegstrecken) die individuelle Betroffenheit von Mautgebühren vermieden werden (darauf deuten etwa auch Befunde zum Verkehrsaufkommen nach Einführung einer City-Maut in London, siehe etwa Ellison et al. 2013). Fasst man die daraus resultierenden Komfortverluste jedoch als nichtmonetäre Kostenkomponente auf (vgl. beispielsweise Brüderl und Preisendörfer 1995), beschneidet dies die Aussagekraft der dargelegten Befunde nicht.

Die zentrale Einschränkung bezieht sich vielmehr darauf, dass es sich bei der Bewertung der vorgelegten Mautmodelle um hypothetische und keine realen Entscheidungen handelte. Die untersuchte abhängige Variable beschreibt die Verhaltensabsicht, eine City-Maut befürworten zu wollen, nicht aber die tatsächliche Befürwortung. Mithin ist fraglich, inwiefern sich eine solche Absicht auch auf tatsächliches Entscheidungsverhalten übertragen lässt. Empirische Untersuchungen zur Validität der in Survey-Experimenten gemessenen Verhaltensabsichten in Bezug auf tatsächliches Verhalten haben teils widersprüchliche Befunde hervorgebracht. Für die vorliegende Untersuchung zentral scheint die sich abzeichnende Erkenntnis, dass in Situationen, in denen kaum normative Erwartungen eines bestimmten Verhaltens bestehen, von einer hohen Übereinstimmung zwischen Verhaltensabsicht und tatsächlichem Verhalten ausgegangen werden kann - zumindest hinsichtlich der Richtung und relativen Stärke von Effekten (Petzold und Wolbring 2019, S. 8). Der möglicherweise dennoch mangelnden externen Validität des Ansatzes sollte durch eine realitätsnahe Ausgestaltung der vorgelegten Beschreibungen möglicher Mautmodelle begegnet werden. Auch die Bewertung verschiedener Mautmodelle, bevor durch die Einführung einer City-Maut tatsächlich Kosten in Form zu leistender Mautgebühren anfallen, korrespondiert mit realen Prozessen politischer Partizipation. Nichtsdestotrotz wären die hier anhand von Verhaltensabsichten untersuchten 
Zusammenhänge in künftigen empirischen Arbeiten auch anhand echter Verhaltensdaten zu prüfen, um die externe Validität der Befunde abzusichern. Zu denken sei dabei beispielsweise an die Untersuchung einer politisch verbindlichen Bürgerbefragung. So könnte anstatt der geäußerten Bereitschaft, das jeweilige Mautmodell zu befürworten, die tatsächliche Befürwortung einer City-Maut in einer Entscheidungssituation untersucht werden, aus der reale Kosten erwachsen (können).

Solange die für reale (Verhaltens-)Entscheidungen relevanten Faktoren nicht mit den hier untersuchten interagieren, sollte aber zumindest die hohe interne Validität des experimentellen Designs gewährleistet bleiben (vgl. Auspurg et al. 2014, S. 44). Zumal es keine Hinweise darauf gibt, dass die in realen Entscheidungen relevanten Faktoren sich derart von den hier untersuchten unterscheiden, bleibt der Kernbefund der vorliegenden Analyse bestehen: Anhand der mikroökonomisch fundierten Teststrategie, wie sie Tutić et al. (2017) vorschlagen, wird die LCH am Beispiel der Befürwortung einer City-Maut in München gestützt.

Während sich die auf der verbesserten Teststrategie basierenden Ergebnisse (trotz nur geringfügig höherer Erklärungskraft der Modelle) als sehr robust erweisen, scheinen die auf der vormals gängigen Teststrategie beruhenden Ergebnisse deutlich auf einzelne Modellierungsentscheidungen zu reagieren. Der viel gravierendere Unterschied zwischen den beiden Teststrategien besteht jedoch darin, dass sie zu inhaltlich unterschiedlichen Schlussfolgerungen führen. Auf Basis der herkömmlichen Teststrategie würde man die LCH verwerfen, obwohl sie sich anhand der verbesserten Teststrategie bewährt.

Insofern stehen die hier vorgelegten empirischen Befunde auch nicht im Widerspruch zu den Ausführungen von Tutić et al. (2017), sondern ergänzen einerseits den noch ausstehenden Test der LCH im engeren Sinn eines Preiseffekts einstellungskonformen Handelns und deuten andererseits einmal mehr darauf hin, dass die theoretisch fundierte Teststrategie dem vormals gängigen Vorgehen auch empirisch vorzuziehen scheint. Es ist jedoch darauf hinzuweisen, dass es sich hierbei um keinen systematischen Vergleich der beiden Teststrategien hinsichtlich ihres generellen empirischen Abschneidens handelt. Eine solche Gegenüberstellung wäre im Rahmen künftiger Forschung zu leisten.

Darüber hinaus scheinen die vorgelegten Befunde auch für die umweltpolitische Debatte um die Konzeption wirksamer Maßnahmen, etwa zur Verringerung der (straBenverkehrsbedingten) Luftschadstoffbelastung in Ballungsräumen, relevant. Neben der breiten Streuung in der Bewertung der hypothetischen Mautmodelle zeigt sich auch das im Rahmen der LCH postulierte Zusammenspiel von Einstellung und Kosten. Aus praktischer Perspektive verweist dies einerseits auf die Bedeutung der langfristigen Entwicklung des Umweltbewusstseins (beispielsweise Franzen und Vogl 2013) und rückt andererseits die Kostenkomponente als möglichen Ansatzpunkt in den Fokus. Aufschlussreich ist, dass sich die vermuteten Zusammenhänge bereits bei der vorgelagerten Bereitschaft zur einstellungskonformen Veränderung (gesetzlicher) Rahmenbedingungen zeigen - noch bevor also tatsächliche Kosten erwachsen. Das mag für die Konzeption (umwelt-)politischer Maßnahmenbündel bedeutend sein, die sowohl öffentlichen Zuspruch erfahren als auch steuerungspolitische Wirkung entfalten (beispielsweise Wicki et al. 2019). Dabei ist im Einzelfall auch das 
Angebot möglicher Handlungsalternativen, inwiefern etwa die (wahrgenommene) Möglichkeit zum Umstieg auf andere Verkehrsmittel besteht, zu berücksichtigen.

Danksagung Wertvolle Anmerkungen zu einer früheren Version des Manuskripts sind Katrin Auspurg, Christian Ganser und Werner Fröhlich sowie 2 anonymen Gutachterinnen oder Gutachtern und der Herausgeberin und den Herausgebern der KZfSS zu verdanken. Ulf Liebe sei für die freundliche Bereitstellung der Analysefiles zu Tutić et al. (2017) gedankt.

Data note Die Studie wurde zusammen mit Katrin Auspurg und Sabine Düval durchgeführt. Für Unterstützung bei der Umsetzung sei zudem den Kolleginnen und Kollegen Christiane Bozoyan, Werner Fröhlich, Christian Ganser, Bettina Pettinger und Michael Rochlitz sowie allen beteiligten studentischen Hilfskräften gedankt. Den Teilnehmerinnen und Teilnehmern der Methoden-Kurse im Wintersemester 2017/18 sei für ihre Mitarbeit an der Konzeption des Fragebogens gedankt. Die Erhebung fand zwischen Mitte Mai und Anfang Juli 2018 statt, wobei einzelne Fragebögen noch bis Ende Juli nacherfasst wurden. Die Studie wurde aus Institutsmitteln finanziert. Analysefiles sind auf Anfrage erhältlich.

Funding Open Access funding provided by Projekt DEAL.

Open Access Dieser Artikel wird unter der Creative Commons Namensnennung 4.0 International Lizenz veröffentlicht, welche die Nutzung, Vervielfältigung, Bearbeitung, Verbreitung und Wiedergabe in jeglichem Medium und Format erlaubt, sofern Sie den/die ursprünglichen Autor(en) und die Quelle ordnungsgemäß nennen, einen Link zur Creative Commons Lizenz beifügen und angeben, ob Änderungen vorgenommen wurden.

Die in diesem Artikel enthaltenen Bilder und sonstiges Drittmaterial unterliegen ebenfalls der genannten Creative Commons Lizenz, sofern sich aus der Abbildungslegende nichts anderes ergibt. Sofern das betreffende Material nicht unter der genannten Creative Commons Lizenz steht und die betreffende Handlung nicht nach gesetzlichen Vorschriften erlaubt ist, ist für die oben aufgeführten Weiterverwendungen des Materials die Einwilligung des jeweiligen Rechteinhabers einzuholen.

Weitere Details zur Lizenz entnehmen Sie bitte der Lizenzinformation auf http://creativecommons.org/ licenses/by/4.0/deed.de.

\section{Anhang}

Tab. 4 Übersicht der im Rahmen der Low-Cost-Hypothese vermuteten Zusammenhänge anhand partieller Ableitungen der Nachfragefunktion (vgl. Tutić et al. 2017, S. 657)

\begin{tabular}{ll}
\hline Vermuteter Zusammenhang & Partielle Ableitung \\
\hline Positiver Effekt der Einstellung & $\frac{\partial x_{a}^{*}\left(\alpha, m, p_{a}\right)}{\partial \alpha}=\frac{m}{p_{a}}>0$ \\
$\begin{array}{l}\text { Negativer Effekt der Kosten einstellungskonfor- } \\
\text { men Handelns }\end{array}$ & $\frac{\partial x_{a}^{*}\left(\alpha, m, p_{a}\right)}{\partial p_{a}}=-\alpha \frac{m}{p_{a}^{2}}<0$ \\
Kostenabhängige Stärke des Einstellungseffekts & $\frac{\partial^{2} x_{a}^{*}\left(\alpha, m, p_{a}\right)}{\partial p_{a} \partial \alpha}=\frac{\partial \frac{m}{p_{a}}}{\partial p_{a}}=-\frac{m}{p_{a}^{2}}<0$ \\
\hline
\end{tabular}


Tab. 5 Dimensionen und Levels der fiktiven Mautmodelle

\begin{tabular}{|c|c|c|c|}
\hline & Dimension & Level & $\Sigma$ \\
\hline 1 & $\begin{array}{l}\text { Verbesserung der } \\
\text { Luftqualität im Stadt- } \\
\text { gebiet München }\end{array}$ & $\begin{array}{l}-\operatorname{Um~} 1 \% \\
-[\mathrm{Um} 5 \%] \\
-\operatorname{Um~} 10 \% \\
-[\mathrm{Um} 15 \%] \\
-\operatorname{Um~} 20 \%\end{array}$ & 5 \\
\hline 2 & $\begin{array}{l}\text { Orte mit besonders } \\
\text { starker Luftverbesse- } \\
\text { rung }\end{array}$ & $\begin{array}{l}\text { - Gesamte Mautzone } \\
\text { - Übermäßig belastete Kreuzungen } \\
\text { - Keine (gleichmäßige Verbesserung im Stadtgebiet) }\end{array}$ & 3 \\
\hline 3 & $\begin{array}{l}\text { Verkehrsaufkommen } \\
\text { im Stadtgebiet }\end{array}$ & $\begin{array}{l}\text { - Unverändert } \\
\text { - Etwas geringer } \\
\text { - Deutlich geringer }\end{array}$ & 3 \\
\hline 4 & $\begin{array}{l}\text { Geltungsbereich der } \\
\text { Maut }\end{array}$ & $\begin{array}{l}\text { - Innerhalb des Altstadtrings (mit Altstadtring) } \\
\text { - Innerhalb des Mittleren Rings (ohne Ring) } \\
\text { - Innerhalb des Mittleren Rings (mit Ring) } \\
\text { - Gesamtes Stadtgebiet (ohne Autobahn) }\end{array}$ & 4 \\
\hline 5 & $\begin{array}{l}\text { Von Maut betroffene } \\
\text { Fahrzeuge }\end{array}$ & $\begin{array}{l}\text { - Alle Fahrzeuge } \\
\text { - Moderat und sehr schadstoffreiche Fahrzeuge (alle } \\
\text { ohne „,blaue Plakette“) } \\
\text { - Sehr schadstoffreiche Fahrzeuge (alle ohne „grüne } \\
\text { Plakette“) }\end{array}$ & 3 \\
\hline 6 & $\begin{array}{l}\text { Mautgebühren pro } \\
\text { Tag }^{\mathrm{a}}\end{array}$ & $\begin{array}{l}-1 € \\
-[3 €] \\
-5 € \\
-[7 €] \\
-10 €\end{array}$ & 5 \\
\hline 7 & $\begin{array}{l}\text { Verwendung der } \\
\text { Mautgebühren }\end{array}$ & $\begin{array}{l}\text { - Förderung des öffentlichen Nahverkehrs } \\
\text { - Schaffung von Grünflächen im Stadtgebiet } \\
\text { - Baumaßnahmen zur Stauvermeidung }\end{array}$ & 3 \\
\hline 8 & $\begin{array}{l}\text { Mautbefreiung für } \\
\text { Anwohner }\end{array}$ & $\begin{array}{l}-\mathrm{Ja} \\
- \text { Nein }\end{array}$ & 2 \\
\hline$\Sigma$ & \multicolumn{2}{|c|}{ Vignettenuniversum $(5 \times 3 \times 3 \times 4 \times 3 \times 5 \times 3 \times 2)$} & 6.200 \\
\hline
\end{tabular}

${ }^{\mathrm{a}}$ Grad der Verbesserung der Luftqualität im Stadtgebiet sowie die Höhe der Maut wurde jeweils bei der Hälfte der Befragten zwischen 3 und bei der anderen Hälfte zwischen 5 möglichen Ausprägungen variiert, um mögliche nichtlineare Effekte besser schätzen zu können (betreffende Ausprägungen in eckigen Klammern)

\section{Literatur}

Apte, Joshua S., Michael Brauer, Aaron J. Cohen, Majid Ezzati und C. Arden Pope. 2018. Ambient PM2.5 Reduces Global and Regional Life Expectancy. Environmental Science \& Technology Letters 5:546-551.

Auspurg, Katrin, und Thomas Hinz. 2015. Factorial Survey Experiments. Thousand Oaks, California: SAGE Publications, Inc.

Auspurg, Katrin, und Ulf Liebe. 2011. Choice-Experimente und die Messung von Handlungsentscheidungen in der Soziologie. Kölner Zeitschrift für Soziologie und Sozialpsychologie 63:301-314.

Auspurg, Katrin, Corinna Frodermann und Thomas Hinz. 2014. Berufliche Umzugsentscheidungen in Partnerschaften. Eine experimentelle Prüfung von Verhandlungstheorie, Frame-Selektion und Low-CostThese. Kölner Zeitschrift für Soziologie und Sozialpsychologie 66:21-50. 
Best, Henning. 2008. Die Umstellung auf ökologische Landwirtschaft. Empirische Analysen zur LowCost-Hypothese des Umweltverhaltens. Kölner Zeitschrift für Soziologie und Sozialpsychologie 60:314-338.

Best, Henning. 2009a. Structural and Ideological Determinants of Household Waste Recycling: Results from an Empirical Study in Cologne, Germany. Nature and Culture 4:167-190.

Best, Henning. 2009b. Kommt erst das Fressen und dann die Moral? Eine feldexperimentelle Überprüfung der Low-Cost-Hypothese und des Modells der Frame-Selektion. Zeitschrift für Soziologie 38:131-151.

Best, Henning, und Thorsten Kneip. 2011. The Impact of Attitudes and Behavioral Costs on Environmental Behavior: A Natural Experiment on Household Waste Recycling. Social Science Research 40:917-930.

Best, Henning, und Clemens Kroneberg. 2012. Die Low-Cost-Hypothese. Theoretische Grundlagen und empirische Implikationen. Kölner Zeitschrift für Soziologie und Sozialpsychologie 64:535-561.

Braun, Norman, und Axel Franzen. 1995. Rationalität und Umweltverhalten. Kölner Zeitschrift für Soziologie und Sozialpsychologie 47:231-248.

Braun, Norman, und Thomas Gautschi. 2011. Rational-Choice-Theorie. Weinheim: Juventa.

Brüderl, Josef, und Peter Preisendörfer. 1995. Der Weg zum Arbeitsplatz: Eine empirische Untersuchung zur Verkehrsmittelwahl. In Kooperatives Umwelthandeln: Modelle, Erfahrungen, Massnahmen, Hrsg. Andreas Diekmann und Axel Franzen, 69-88. Chur/Zürich: Verlag Rüegger AG.

Burke, William J. 2009. Fitting and Interpreting Cragg's Tobit Alternative using Stata. The Stata Journal 9:584-592.

Cragg, John G. 1971. Some Statistical Models for Limited Dependent Variables with Application to the Demand for Durable Goods. Econometrica 39:829-844.

Derksen, Linda, und John Gartrell. 1993. The Social Context of Recycling. American Sociological Review 58:434-442.

Diekmann, Jochen. 1998. Umwelt, Ökonomik und empirische Sozialforschung. Bemerkungen zum interdisziplinären Diskurs. In Umwelt und empirische Sozial- und Wirtschaftsforschung, Hrsg. Jürgen Schupp und Gert Wagner, 187-198. Berlin: Duncker und Humblot.

Diekmann, Andreas, und Peter Preisendörfer. 1998. Umweltbewußtsein und Umweltverhalten in Lowund High-Cost-Situationen. Eine empirische Überprüfung der Low-Cost-Hypothese. Zeitschrift für Soziologie 27:438-453.

Diekmann, Andreas, und Peter Preisendörfer. 2003. Green and Greenback. The behavioral effects of environmental attitudes in low-cost and high-cost situations. Rationality and Society 15:441-472.

Ellison, Richard B., Stephen P. Greaves und David A. Hensher. 2013. Five Years of London's Low Emission Zone: Effects on Vehicle Fleet Composition and Air Quality. Transport Research Part D 23:25-33.

Findley, Michael G., Brock Laney, Daniel L. Nielson und J. C. Sharman. 2017. External Validity in Parallel Global Field and Survey Experiments on Anonymous Incorporation. The Journal of Politics 79:856-872.

Franzen, Axel, und Dominikus Vogl. 2013. Two decades of measuring environmental attitudes: A comparative analysis of 33 countries. Global Environmental Change 23:1001-1008.

Hainmueller, Jens, Dominik Hangartner und Teppei Yamamoto. 2015. Validating Vignette and Conjoint Survey Experiments against Real-World Behavior. Proceedings of the National Academy of Sciences 112:2395.

Keuschnigg, Marc, und Fabian Kratz. 2018. Thou Shalt Recycle: How Social Norms of Environmental Protection Narrow the Scope of the Low-Cost Hypothesis. Environment and Behavior 50:1059-1091.

Lelieveld, Jos, Klaus Klingmüller, Andrea Pozzer, Ulrich Pöschl, Mohammed Fnais, Andreas Daiber und Thomas Münzel. 2019. Cardiovascular Disease Burden from Ambient Air Pollution in Europe Reassessed Using Novel Hazard Ratio Functions. European Heart Journal 40:1590-1596.

Liebe, Ulf. 2007. Zahlungsbereitschaft für kollektive Umweltgüter. Soziologische und ökonomische Analysen. Wiesbaden: VS Verlag für Sozialwissenschaften.

Liebe, Ulf, und Benedikt Jahnke. 2017. Giving More to Humans than to Animals in Need? A Behavioral Measure of Animal-Human Continuity in Large-scale Surveys. Journal of International Society for Anthrozoology 30:249-262.

Mas-Collel, Andreu, Michael D. Winston und Jerry R. Green. 1995. Microeconomic theory. New York: Oxford University Press.

Mayerl, Jochen. 2010. Die Low-Cost-Hypothese ist nicht genug. Eine Empirische Überprüfung von Varianten des Modells der Frame-Selektion zur besseren Vorhersage der Einflussstärke von Einstellungen auf Verhalten. Zeitschrift für Soziologie 39:38-59. 
Neumann, Robert, und Guido Mehlkop. 2018. Umweltentscheidungen als Wechselspiel von Einstellungen, Handlungskosten und situativer Rahmung - ein empirischer Theorienvergleich mit Daten des GESIS Panels. Zeitschrift für Soziologie 47:101-118.

Petzold, Knut, und Tobias Wolbring. 2019. What Can We Learn from Factorial Surveys About Human Behavior? A Validation Study Comparing Field and Survey Experiments on Discrimination. Methodology: European Journal of Research Methods for the Behavioral and Social Sciences 15:19-30.

Pope, C. Arden, und Douglas W. Dockery. 2006. Health Effects of Fine Particulate Air Pollution: Lines that Connect. Journal of Air \& Waste Management Association 56:709-742.

Preisendörfer, Peter. 2000. Strukturell-situationale Gegebenheiten als Bestimmungsfaktoren der Verkehrsmittelwahl. Soziale Welt 51:487-501.

Rauhut, Heiko, und Ivar Krumpal. 2008. Die Durchsetzung sozialer Normen in Low-Cost und High-Cost Situationen. Zeitschrift für Soziologie 37:380-402.

Schultz, P. Wesley, und Stuart Oskamp. 1996. Effort as a Moderator of the Attitude-Behavior Relationship: General Environmental Concern and Recycling. Social Psychology Quarterly 59:375-383.

Tutić, Andreas, Thomas Voss und Ulf Liebe. 2017. Low-Cost-Hypothese und Rationalität. Eine neue theoretische Herleitung und einige Implikationen. Kölner Zeitschrift für Soziologie und Sozialpsychologie 69:651-672.

Umweltbundesamt. 2017. Daten zur Umwelt 2017: Indikatorenbericht. Dessau-Roßlau: Umweltbundesamt.

Varian, Hal R. 1992. Microeconomic analysis. New York: Norton.

Wicki, Michael, Lukas Fesenfeld und Thomas Bernauer. 2019. In search of politically feasible policypackages for sustainable passenger transport: insights from choice experiments in China, Germany, and the USA. Environmental Research Letters 14.

Fabian Thiel 1992, M.A., wissenschaftlicher Mitarbeiter am Institut für Soziologie der Ludwig-Maximilians-Universität München. Forschungsinteressen: Methoden der empirischen Sozialforschung, Umweltsoziologie. Veröffentlichungen: Different samples, different results? How sampling techniques affect the results of field experiments on ethnic discrimination. Research in Social Stratification and Mobility 65, 2020 (mit K. Auspurg und A. Schneck). 